\title{
Frequency shift of the minimal magnitude of reflection coefficient in antenna array
}

\author{
Ferdinand Vavrík, Jozef Hallon*
}

\begin{abstract}
The paper present a study of frequency shift of the reflection coefficient minimal absolute value dependence on antenna spacing in linear and circular arrays. The spacing of CB-band monopole antenna was in the range of 0.015 to 0.12 multiples of the antenna working wavelength. Number of elements in the antenna arrays ranged from two to four. Frequency dependence of minimal absolute value of the reflection coefficient was obtained by simulations and also experimentally confirmed in anechoic chamber. The research showed two facts. First, mutual impedance has the higher impact when antenna spacing is comparable with the reactive near field region. Second, circular antenna arrays are a better choice in applications for a small number of antennas with that spacing.
\end{abstract}

K e y w ords: antenna array, reflection coefficient, mutual impedance

\section{Introduction}

Reflection coefficient $\mathcal{S}_{11}$ for antenna in free space is expressed as following

$$
\mathcal{S}_{11}=\frac{\mathcal{Z}_{A}-\mathcal{Z}_{0}}{\mathcal{Z}_{A}+\mathcal{Z}_{0}}
$$

where $\mathcal{Z}_{A}$ is impedance of the load and $\mathcal{Z}_{0}$ is characteristic impedance of the transmission line [1]. The impedance of the antenna positioned among other antennas, elements or obstacles, depends upon self-impedance (impedance measured at the driven antenna without any obstacles or other antennas) and the mutual impedance among the driven antenna and other antennas or obstacles [2]. Therefore, the mutual impedance has influence on the reflection coefficient, as shown in the following equation

$$
\mathcal{S}_{11}=\frac{\left(\mathcal{Z}_{11}-\mathcal{Z}_{0}\right)\left(\mathcal{Z}_{22}+\mathcal{Z}_{0}\right)-\mathcal{Z}_{12}^{2}}{\left.\mathcal{Z}_{11}+\mathcal{Z}_{0}\right)\left(\mathcal{Z}_{22}+\mathcal{Z}_{0}\right)-\mathcal{Z}_{12}^{2}}
$$

where $\mathcal{Z}_{11}$ and $\mathcal{Z}_{22}$ are self-impedances of antenna 1 and 2 in a simple two antenna array, $\mathcal{Z}_{0}$ is the characteristic impedance of transmission lines connecting all elements in the array and $\mathcal{Z}_{12}$ is the mutual impedance between the two antennas [3].

The mutual impedance is inversely proportional to the square of the distance between the respective antennas [3]. Thus, the reflection coefficient of a single antenna in an array can be considered as a suitable parameter to express the changes in performance of the antenna array induced by changing the antenna spacing $d$.

The mutual impedance is phenomena of the near field region [3]. This region can be divided to reactive near field region, close surrounding of the antenna and radiating near field region following the previous. The boundary of reactive near field region $R_{1}$ is

$$
R_{1}=0.62 \sqrt{\frac{D^{3}}{\lambda}}
$$

where $\lambda$ is working wavelength of the antenna and $D$ is the largest dimension of the antenna [2]. The end boundary of radiating near field region $R_{2}$ is

$$
R_{2}=\frac{2 D^{2}}{\lambda}
$$

where $\lambda$ and $D$ have the same meaning as in (3).

We present theoretical and experimental findings concerning the frequency $f_{m}$, at which the magnitude of reflection coefficient is minimal, and the shift of this frequency while changing the antenna spacing $d$ in a linear and circular array. Results of some works by different authors do show this effect, but without any further comments [4]. To our best knowledge, there is no other work that would concentrate its efforts primarily on studying this effect.

\section{Experiment}

Four $1.5 \mathrm{~m}$ length monopole antennas manufactured for CB band (26-28 MHz) were used for the experiment. For these antennas the boundary $R_{1}$ is $0.928 \mathrm{~m}$ and $R_{2}$ is $1.5 \mathrm{~m}$. The two ports vector network analyzer was used for measuring the reflection coefficient in anechoic chamber.

The range of antenna spacing $d$ has been from $0.015 \lambda$ to $0.12 \lambda$. This corresponds to approximately 15 to $130 \mathrm{~cm}$. The spacing $15 \mathrm{~cm}$ is the smallest possible distance, because of dimensions of antenna pedestal.

* Slovak University of Technology in Bratislava, Faculty of Electrical Engineering and Information Technology, Bratislava, Slovakia, ferdinand.vavrik@stuba.sk; jozef.hallon@stuba.sk 

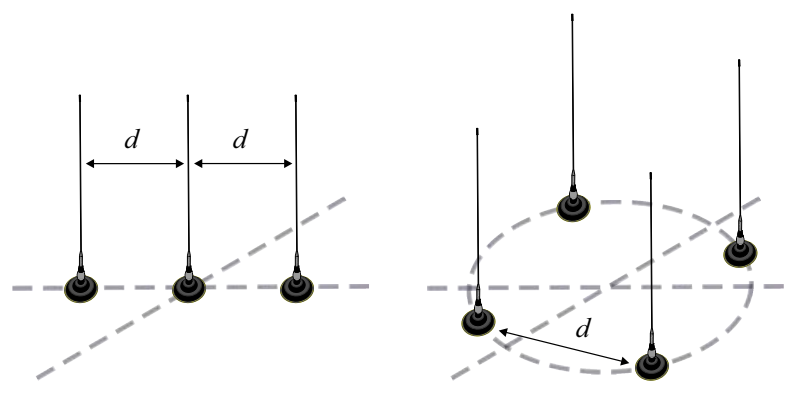

Fig. 1. The schematic of basic types of antenna arrays (linear and circular), $d$-antenna distance, which was the free parameter during our measurements and simulations

Five configurations of antenna array has been measured for two basic types of antenna arrays (Fig. 1). The first configuration consisted of two antennas. This configuration can be treated as both a linear or circular array, since the two antennas can be viewed as placed on a circle. For this reason, the data obtained from this configuration were used as a reference case, to which the results of other configurations were normalized. The other configurations consisted of three and four elements, in both linear and circular arrays.

During a measurement, the data were collected from a single antenna, while others were terminated by $50 \Omega$ load. Measurement was performed at each position in an array, while taking advantage of symmetry, eg in a threeelement linear array, data were collected from the center and one of the edge antennas, in two measurements. For a circular array the positions are equivalent.

To account for the variations in the reflection coefficient caused by slight manufacturing-induced differences in the impedances of the antennas, their positions for each measurement were systematically permuted. Therefore, several measurement series, each consisting of measurements with varying distance and positional permutations, were performed for each array configuration.

The experimental results - magnitude of reflection coefficient in 20 to $30 \mathrm{MHz}$ frequency range are in Fig. 2.

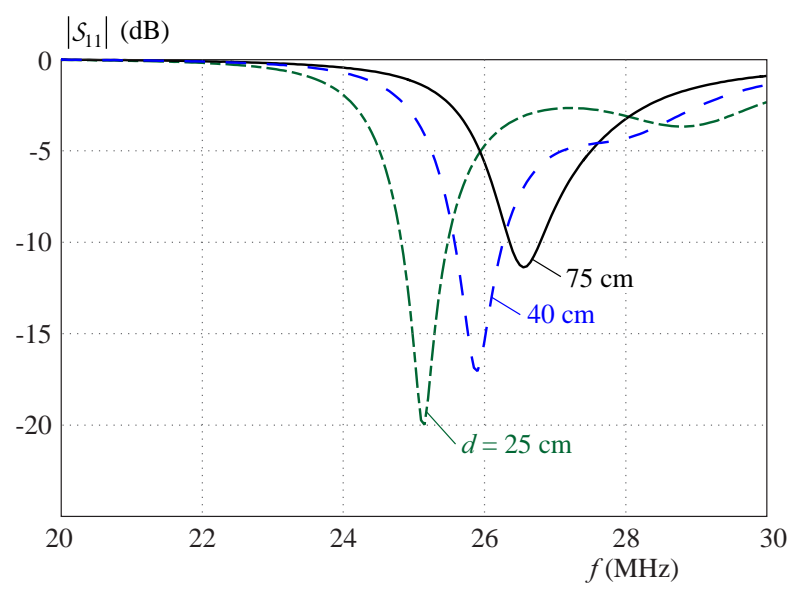

Fig. 2. Frequency dependence of magnitude of the reflection coefficient on antenna spacing $d$ in a three element circular array
For each configuration and different antenna distances, the minima of the reflection coefficient magnitude were located, obtaining a series of frequency values created by positional permutations. These were subsequently averaged and their standard deviations calculated, thus creating a frequency-distance plot. Experimental results for each array configuration are presented in Fig. 3 to Fig. 13.

\section{Simulations}

The simulation software Altair FEKO was used to calculate dependencies of the reflection coefficient curves in respect to antenna spacing in arrays, in the frequency range of 20 to $30 \mathrm{MHz}$. Altair FEKO uses the method of moments (MoM) for calculation of the coupling analysis [5], which best suits our interest.

We modeled four antennas in accordance with properties of the antennas used in the experiment. The same array configurations as in the experiment, were used.

The input values and parameter variation for the simulations were the same as for the experiments. The only difference was one additional parameter, which is the deflection of the top of the antenna in respect to the bottom of the antenna. The experimental antennas were not perfectly vertical, with a maximum of $4 \mathrm{~cm}$ of deflection, this value was used in simulations. The deflection was modeled in three directions. First in direction towards to the data collection antenna, second in direction away from the data collection antenna and finally perpendicular to the line connecting the deflected antenna and the data collection antenna.

Several simulation series were performed for each array configuration, taking into account all combinations of the relevant input parameter, mimicking the systematic approach of the experiments. Data processing was also identical. We selected the frequency positions of the minima of the calculated reflection coefficient curves. Data points in a series, calculated for an array configuration and antenna distance, were averaged and their standard deviations were calculated. The resulting simulated frequency-

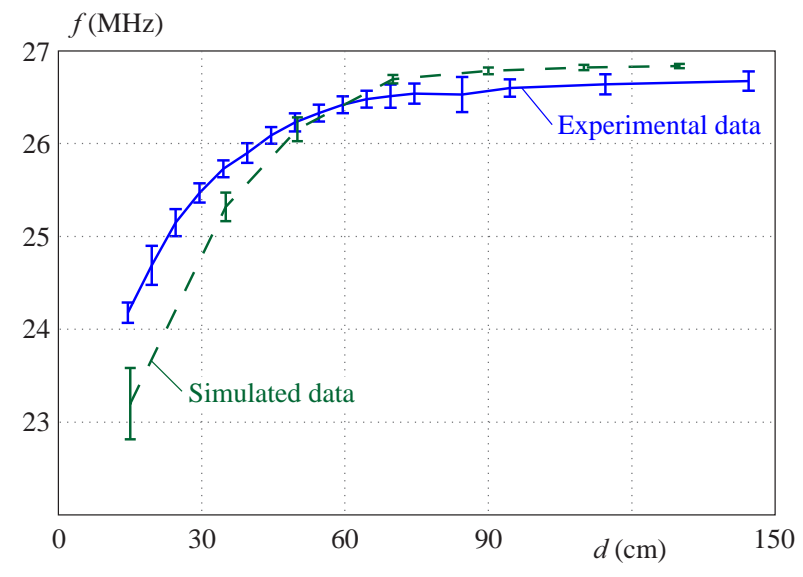

Fig. 3. Frequency dependence of $f_{m}$ on antenna spacing $d$ for the reference 2-antenna array 


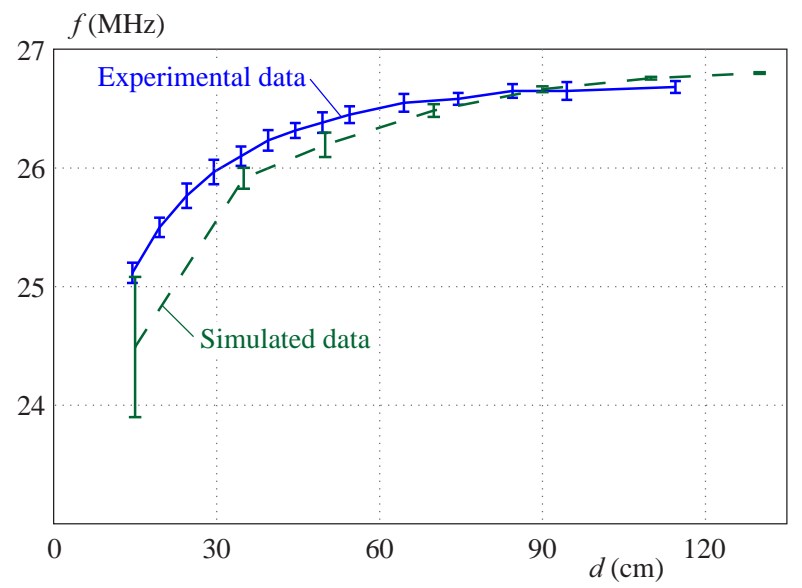

Fig. 4. Frequency dependence of $f_{m}$ on antenna spacing $d$ for 3element linear antenna array with data collection antenna on the edge of the array

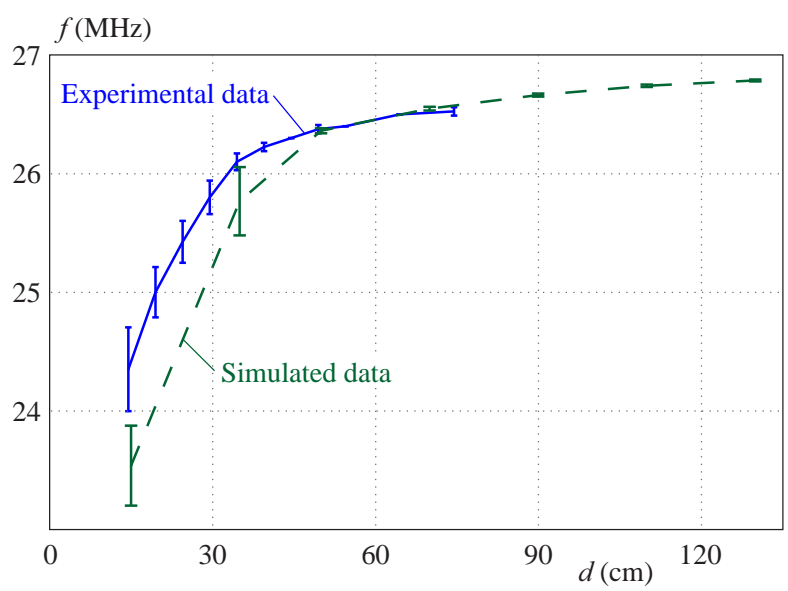

Fig. 6. Frequency dependence of $f_{m}$ on antenna spacing $d$ for 4element linear antenna array, with data collection antenna at the edge of the array

distance curves are presented for each array configuration in Fig. 3 to Fig. 13.

\section{Measured and simulated data}

The frequency dependence of reflection coefficient magnitude, shown in the Fig. 2, has been measured for circular antenna array with three antennas. It shows the shift of magnitude minima at $f_{m}$ towards the lower frequencies with decreasing antenna distance.

The results of the measurement and simulation of the reference antenna array consisting of two antennas are in Fig. 3. As could be seen in Fig. 3, the significant change of frequency $f_{m}$ is in interval of spacing $d$ from 0.15 to $0.9 \mathrm{~m}$. This corresponds to dimension of reactive near field region.

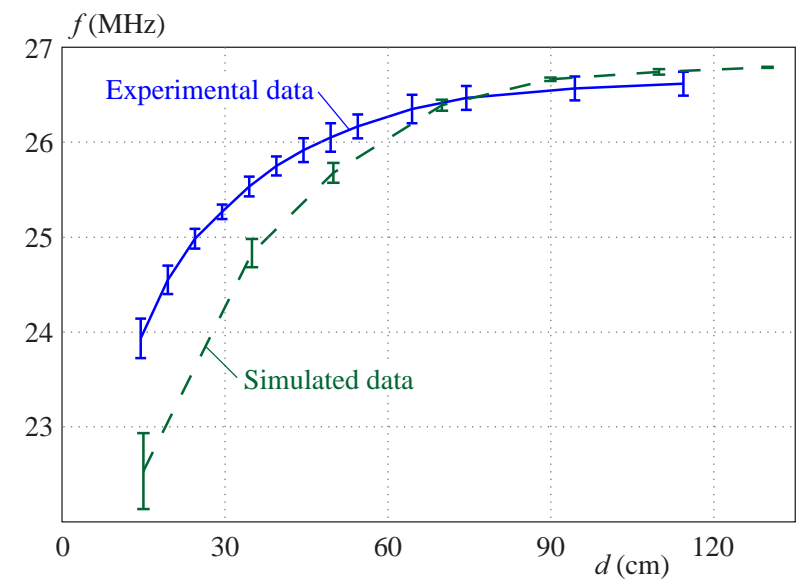

Fig. 5. Frequency dependence of $f_{m}$ on antenna spacing $d$ for 3 element linear antenna array, with data collection antenna in the center of the array

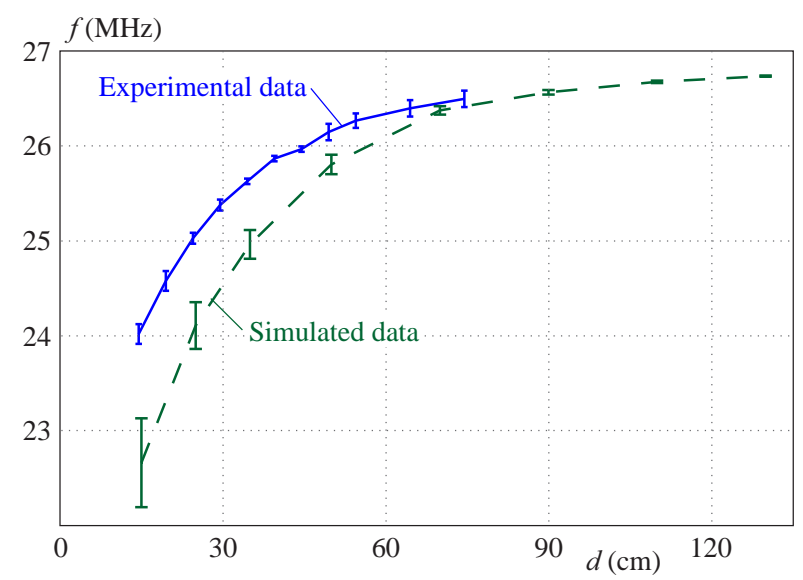

Fig. 7. Frequency dependence of $f_{m}$ on antenna spacing $d$ for 4-element linear antenna array, with data collection antenna in position next to the edge of the array

\subsection{Linear arrays}

The data collection antenna was placed in 3-element linear antenna array at its edge (Fig. 4) and in its center respectively (Fig. 5). As could be seen in Fig. 4 and Fig. 5, the frequency dependence of $f_{m}$ "point" shows the same trend as in the reference array. However, values in Fig. 4 for spacing $d$ from $15 \mathrm{~cm}$ to $40 \mathrm{~cm}$ is higher than it is in the reference array.

The position of data collection antenna in 4-element linear antenna array was alternatively at the edge (Fig. 6) and next to the edge of array (Fig. 7). The maximum value of spacing $d$ for 4-element linear antenna array was $75 \mathrm{~cm}$, because of anechoic chamber dimensions. This had practically no impact on results, because values for distance $d=75 \mathrm{~cm}$ have lesser differences than for $d=$ $90 \mathrm{~cm}$. Frequency dependences of $f_{m}$ for these two cases have also the same trend as in reference array. However, the values in Fig. 6 show faster decreasing when distance $d$ is smaller than $35 \mathrm{~cm}$. 


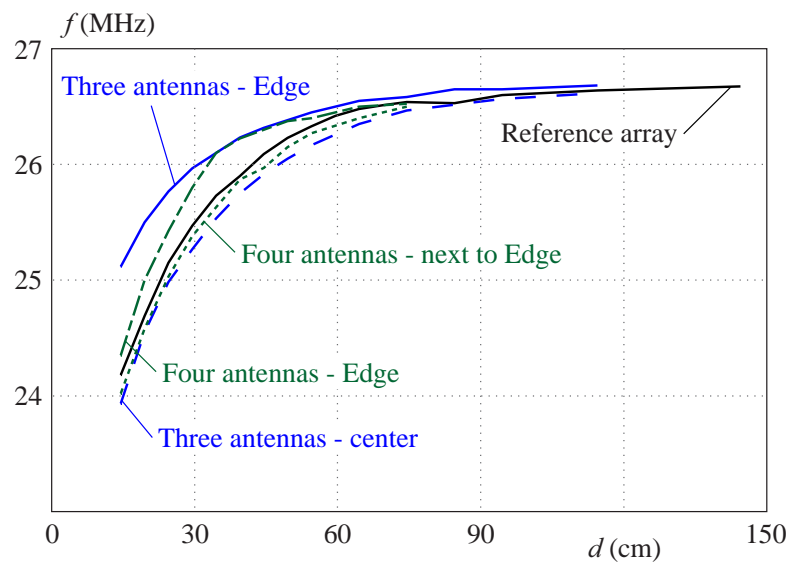

Fig. 8. Comparison of experimental data of 3 and 4-element linear antenna arrays with reference array

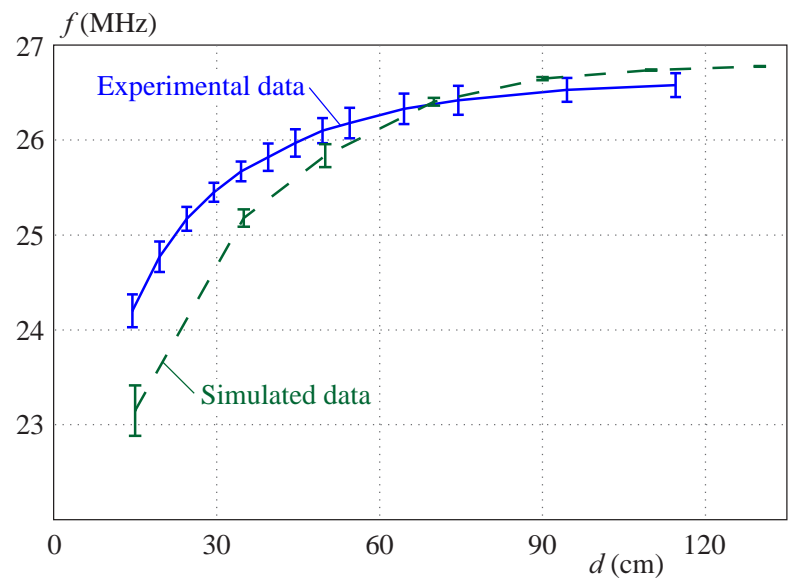

Fig. 10. Frequency dependence of $f_{m}$ point with respect to antenna spacing $d$ for 3-element circular antenna array

The comparison of measured data of 3 and 4-element linear antenna array with reference antenna is in Fig. 8. As could be seen, the data measured at the edge of arrays has higher values for spacing $d$ from 15 to $50 \mathrm{~cm}$ than in other cases. This was as expected for the edge antennas. The simulation data shows the same trends as experimental data in Fig. 9.

\subsection{Circular arrays}

The position of data collection antenna in a circular antenna array is not specific as before. The experimental and simulated data of 3-element circular antenna array are in Fig. 10. As shown, the frequency dependence of $f_{m}$ point exhibits the same trend as it is in the reference array.

The experimental and simulated data of 4-element circular antenna arrays showed in Fig. 11 are almost the same as data in Fig. 10. This is clear in Fig. 12 and 13 , presenting comparison of experimental and simulated data of 3 and 4-element circular antenna array with reference array.

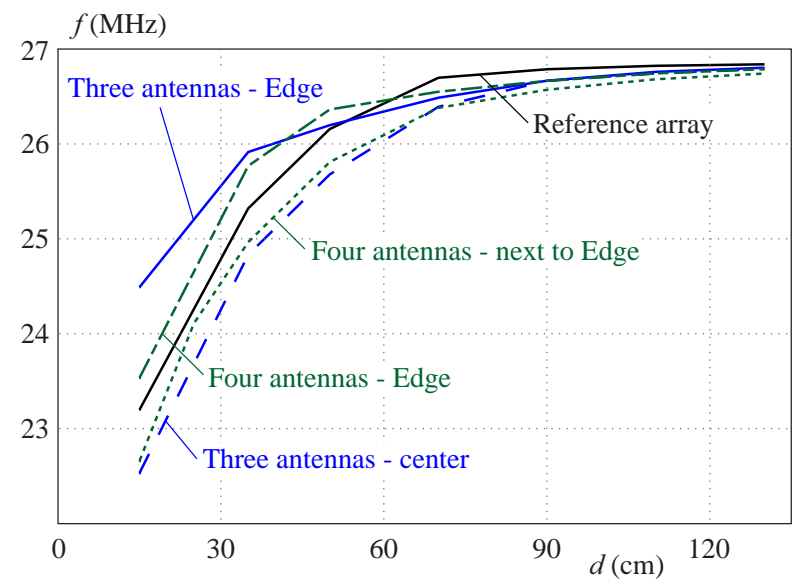

Fig. 9. Comparison of simulation data of 3 and 4-element linear antenna arrays with reference array

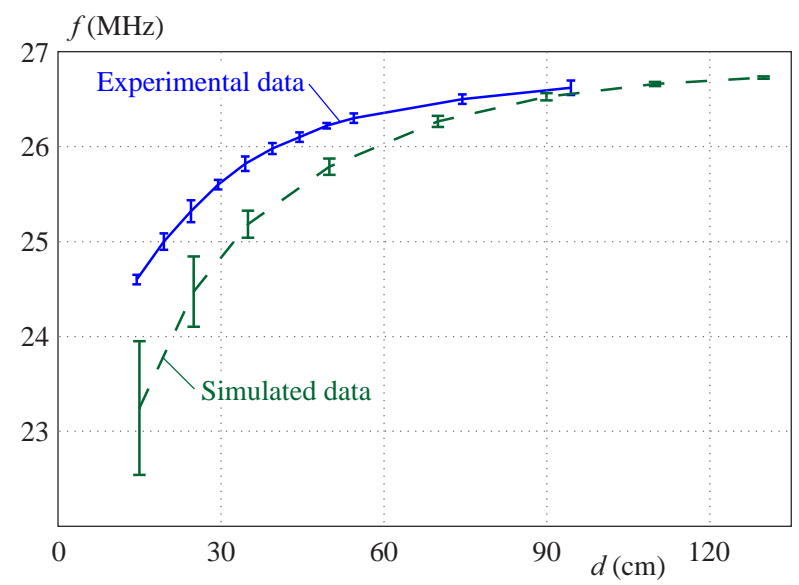

Fig. 11. Frequency dependence of $f_{m}$ point with respect to antenna spacing $d$ for 4-element circular antenna array

As could be seen in Figs. 12 and 13, there is almost no difference in data of antennas in circular arrays and the reference array.

\section{Conclusion}

The frequency shift of the minimal magnitude of reflection coefficient (point $f_{m}$ ) due to changed antenna spacing $d$ has been studied. As the experimental and simulated data showed, the position of $f_{m}$ point on the frequency scale depends on the antenna spacing $d$. As the antenna spacing decreases, the position of the $f_{m}$ point is shifting towards lower frequencies. This shift is significant when spacing $d$ is comparable with the dimension of reactive near field region. The frequency shift of $f_{m}$ in radiating near field region was negligible. So contention: the mutual impedance is phenomenon of the near field.

The differences in standard deviation of simulated data are caused by not perfectly matched simulation model of antennas with the real antennas. However the comparison of the experiments and simulations showed the same 


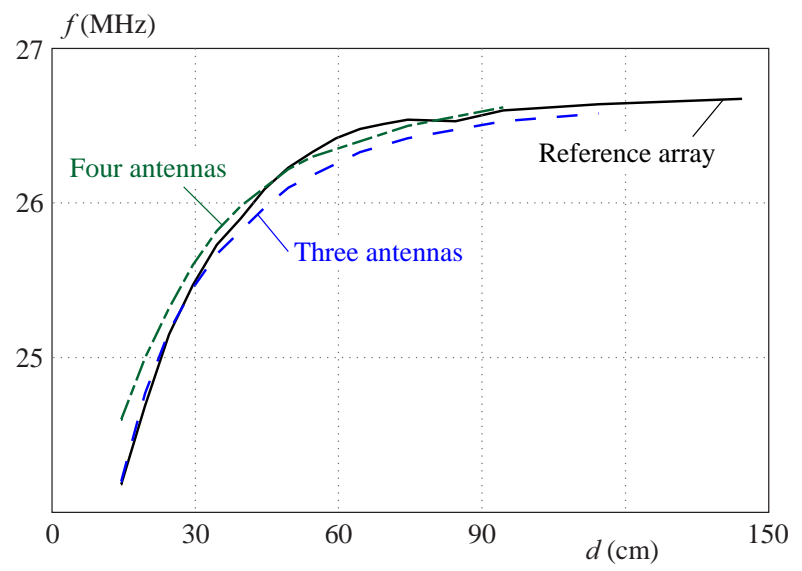

Fig. 12. Comparison of the experimental data of 3 and 4-element circular antenna array with reference array

trend in antenna behavior in respect to the antenna spacing $d$. It could be stated, the simulations were confirmed by experimental data.

The presented results showed, that frequency shift of $f_{m}$ point of antenna placed among others is the same as in reference antenna array. The frequency shift of $f_{m}$ point of edge antenna is lower than for antennas placed inside of antenna array. It is caused by asymmetrical configuration of the neighboring antennas. Antennas inside an circular array have symmetrically placed neighbors. The closest antennas have decisive impact. This could be seen in comparison of 3-element linear antenna array with data collected antenna in the center and 4-element linear antenna array with data collected antenna in position next to the edge of antenna array. The data collecting antenna has two closest antennas except for 4-element linear antenna array, where is one extra antenna. Despite of this extra antenna, the frequency shift of $f_{m}$ point in this two cases is the same.

The presented results also showed that all antennas in circular antenna arrays have the same frequency shift of $f_{m}$ point, due to nonexistence of "edge" antennas. This leads to a conclusion that circular antenna arrays are a better choice for applications of small number of antennas with antenna spacing comparable with dimensions of the "reactive near field region". The edge effect has impact on radiation pattern of the whole antenna array.

\section{Acknowledgements}

Work presented in this paper was supported by the Slovak Research and Development Agency APVV, No. APVV-15-0062 and No. APVV-15-0257.

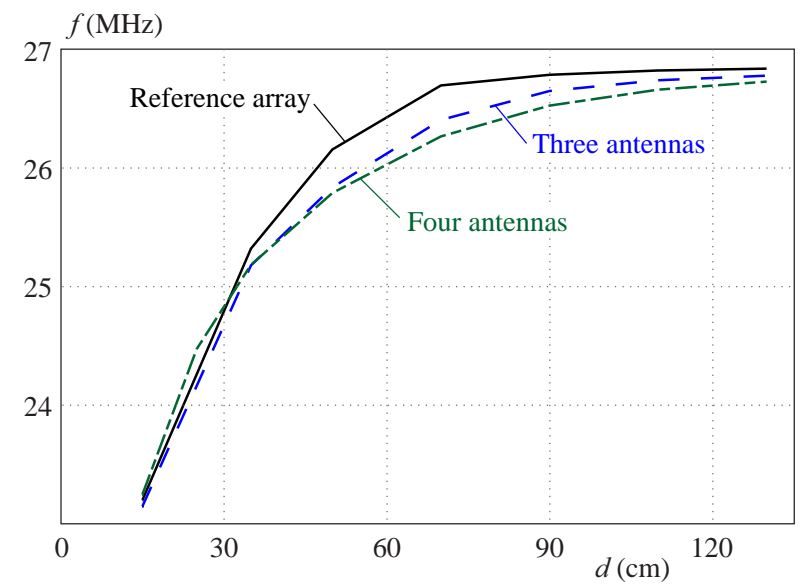

Fig. 13. Comparison of the simulated data of 3 and 4-element circular antenna array with reference array

\section{REFERENCES}

[1] T. A. Milligan, Modern antenna design, 2nd edition, 2005.

[2] C. A. Balanis, Antenna Theory: Analysis and Design, 3rd edition, Canada, 2005.

[3] W. L. Stutzman and G. A. Thiele, Antenna Theory and Design, 3rd edition, pp. 306-308, 2012.

[4] A. Osama and A. R. Sebak, "Mutual Coupling Effect on Ultrawideband Linear Antenna Array Performance", International Journal of Antennas and Propagation, vol. 2011, Article ID 142581 .

[5] Numerical Methods in FEKO. Online: https:/www.altair.com /RelatedCaseStudy.aspx?id=7172.

Received 13 December 2018

Ferdinand Vavrík was born in Ilava, Slovakia. He is a PhD student at the Faculty of Electrical Engineering and Information Technology, Slovak University of Technology in Bratislava. He received the MSc. Degree from the Faculty of Electrical Engineering and Information Technology, Slovak University of Technology in Bratislava in 2015.

Jozef Hallon was born in Bratislava, Slovakia. He received the MSc and PhD degree at the Faculty of Electrical Engineering and Information Technology, Slovak University of Technology in Bratislava in 1987 and 2010, respectively. During the years 1987 - 1992 he had been working as a research worker at Slovak Academy of Science. Since 1992 he has been working as a research worker at the Faculty of Electrical Engineering and IT in the EMC group of the Institute of Electrical Engineering. His research interests are in the area of measuring methods in EMC, immunity tests of electrical devices and consulting in EMC. 